\section{Visual outcomes and anatomic changes after sub-threshold micropulse yellow laser (577-nm) treatment for chronic central serous chorioretinopathy: long-term follow-up}

A Arsan, HS Kanar and A Sonmez
Turkey Ophthalmology

Society, Istanbul, Turkey

Correspondence:

HS Kanar, Fatih Sultan Mehmet Training and Research Hospital, Ali Nihat Tarlan Caddesi No: 72/20, 34800 Istanbul, Turkey Tel: +905327229188; Fax: +904722157352 . E-mail: hselensonmez@ hotmail.com

Received: 5 June 2017 Accepted in revised form: 30 October 2017

Published online: 5 January 2018

\begin{abstract}
Purpose To analyze the long-term efficacy of $577 \mathrm{~nm}$ sub-threshold micropulse yellow laser (SMYL) in the treatment of chronic central serous chorioretinopathy (CCSC) and to evaluate the anatomic outcome, visual results and safety profile of the treatment. Patients and methods This prospective study assessed 39 eyes of 39 patients with nonresolving CCSC lasting more than three months. All eyes were treated by using $577 \mathrm{~nm}$ SMYL system with $\mathbf{5 \%}$ duty cycle (DC) and each patients was monitored monthly. The main outcome measures were best-corrected visual acuity (BCVA), contrast sensitivity (CS) and subretinal fluid (SRF) height, central macular thickness (CMT), central macular volume (CMV), total macular volume (TMV), and subfoveal choroidal thickness (SFCT) measured by spectral domain optical coherence tomography (SDOCT).

Results The median follow-up time period was $17.82 \pm 0.42(13-23$ months) months. The BCVA was improved significantly at final follow-up in comparison of baseline visit $(P<0.01)$ in 35 eyes $(89.7 \%)$ and in 4 eyes (10.3\%) was stable. The median CMT, CMV, TMV before treatment was $369 \mu \mathrm{m}, 0.30 \mathrm{~mm}^{3}$, and $9.86 \mathrm{~mm}^{3}$, in comparison to $250 \mu \mathrm{m}$, $0.19 \mathrm{~mm}^{3}$, and $8.76 \mathrm{~mm}^{3}$ at final follow-up, respectively ( $P<0.01$ for all these parameters). Initial median SFCT was recorded as $364 \mu \mathrm{m}$ and $342 \mu \mathrm{m}$ at the final follow-up $(P<0.001)$. Discussion Results suggest that SMYL treatment is an effective method as response was rapid and procedure is safe to manage the non-resolving CCSC eyes.
\end{abstract}

Eye (2018) 32, 726-733; doi:10.1038/eye.2017.293; published online 5 January 2018

\section{Introduction}

Central serous chorioretinopathy (CSC) is a disease of neurosensory retinal detachment at the posterior pole caused by leakage of fluid through the decompensated retinal pigment epithelium (RPE) into the subretinal space. Men are more prone to CSC than women, and the average age of affected patients reported in recent large studies has ranged from 45 to 51 year. ${ }^{1}$ Patients mostly complain of loss of visual function including blurred vision with a relative central scotoma and metamorphopsia, possibly associated with dyschromatopsia, micropsia, and a decrease of contrast sensitivity. ${ }^{2}$ The natural course of acute form of CSC is benign. ${ }^{3}$ A large proportion of CSC cases resolve spontaneously; however, up to $20 \%$ of patients may have persistent SRF and visual disturbances lasting several months. ${ }^{4}$ When SRF persists longer than three or four months, the term chronic CSC (CCSC) is generally used. Some authors have defined chronicity as presence of persistent fluid for at least 6 months, whereas recent clinical trials have used three-month time frame as the standard. ${ }^{5}$ Persistency of the SRF may result subretinal fibrin accumulation, RPE atrophy (RPEA), neurosensory retinal atrophic changes, or choroidal neovascularization. ${ }^{6}$

Recurrences in the same or fellow eyes occur in $33-50 \%$ of cases, which may lead to visual dysfunction and could require starting of treatment. Patients with frequent recurrences or chronic neurosensory retinal detachment may 
develop further visual dysfunctions. ${ }^{7}$ Clinicians do not only use the classification of CSC to define the term; classification also seems to be more helpful for informing the decision to start treatment. The goals of CSC treatments are to induce reattachment of the neurosensory retina, improve or maintain visual acuity, and avoid recurrences and loss of RPE.7,8

Photodynamic therapy (PDT), various forms of laser photocoagulation, intravitreal anti-vascular endothelial growth factor (anti-VEGF) agents, and systemic antimineralocorticoid drugs are some of the treatment options but they may have some undesirable side effects, including central or paracentral scotomas, contrast sensitivity loss, retinal distortion, choroidal neovascularization (choroidal ischemia, RPEA, endopthalmitis), and systemic side effects. ${ }^{9,10}$

When the conventional laser (CL) energy is emitted, ocular chromophores absorb the laser energy and convert it into heat that spreads forward to adjacent locations where there is no light absorption. Mainster summarized some principles for limiting retinal damage by changing wavelength, spot size, retinal irradiance, and pulse duration or varying clinical endpoints can minimize the effects of the laser energy. ${ }^{11,12}$ There is also a recent study comparing efficacy of CL and SMYL treatment in CCSC. They observed equivalent therapeutic effects but all eyes in the CL treated group had RPE damage. ${ }^{13}$

Newer laser treatment modalities for various retinal diseases include sub-threshold micropulse lasers (SML), which do not result in photo-thermal elevation. SMLs are designed to avoid visible burn endpoints. They offer the advantage of precise control and spatial confinement of laser lesions to the RPE layer. Moreover, SMLs induce a biological response that promotes the recovery and restoration of the outer blood-retinal barrier, and ultimately, the resorption of the subretinal fluid. These protocols produce only sublethal thermal elevations, with effects that are invisible during treatment and remain so thereafter. ${ }^{14}$ In this laser modalities, the whole-pulse duration is called a pulse envelope, which is divided into 100 micropulses, and each micropulse has 'ON' (active) and 'OFF' (off) time, with a ratio depending on the duty cycle (the ratio between the on time and whole micropulse on and off time-DC). DC is adjustable by the surgeon, thus controlling the intensity and the heat spread. ${ }^{15,16}$

There are limited studies, including mainly $810 \mathrm{~nm}$ SML and some $577 \mathrm{~nm}$ SML, which have mainly shown short-term effects in follow-up visits with a restricted number of eyes. ${ }^{17-19}$ In this study, we aimed to show the long-term efficacy of $577 \mathrm{~nm}$ yellow wavelength SML (SMYL) in the treatment of CCSCR cases and evaluate the anatomic outcomes, visual results, OCT parameters, duration of starting treatment activity, recurrences, and safety profile of the treatment.

\section{Materials and methods}

Thirty-nine eyes of 39 patients who were diagnosed with CCSC or recurrent CSC at the Fatih Sultan Mehmet Training and Research Hospital from October 2014 through March 2017 were enrolled. This prospective study adhered to the ethical standards in the Declaration of Helsinki. The medical histories of the patients and the types of therapies, if any, that had been applied previously (focal laser, PDT, anti-VEGF agents) were noted. All eyes underwent initial assessment by measuring the BCVA using Snellen chart, CS testing with the Pelli-Robson chart, 90 diopter preset lens slit-lamb biomicroscopy. The inclusion criteria were as follows: (1) symptomatic CSC of 3 months or greater, (2) CSC with SRF involving the fovea documented by SD-OCT, and (3) patients who were eligible for monthly visit at least one year. Exclusion criteria were as follows: (1) patients who had a history of comorbide ocular conditions, (2) patients who had received anti-VEGF treatment within 6 months and conventional laser or PDT in the past one year before SMYL treatment, or (3) patients who were not followed up over 12 months after the start of SMYL treatment. Patients with serous macular detachment of the neurosensory retina on SD-OCT (Nikon RS-3000, Japan) and with a single or multiple active leakage sites and if any RPE changes on FA were diagnosed as CSC. All patients had undergone ICGA before treatment to ascertain the CSC diagnosis, and again at 12 month to determine any side effects such as choroidal neovascularization.

CMT, CMV (0.3 $\mathrm{mm}^{3}$ central area of macula), TMV, and SFCT values were measured by SD-OCT for all eyes. SRFH was measured manually between the outer segment of the photoreceptor layer and the apical face of the RPE layer. All OCT measurements were performed by the same technician. In addition, all choroidal thickness data were assessed by the same ophthalmologist, using enhanced-depth imaging (EDI) scans, as the axial distance from the RPE to the outer choroid/sclera interface. These examinations were applied to all participants during each monthly visit. BCVA was converted to logarithm of the minimum angle of resolution (logMAR) units for statistical analysis.

In this study, a 577-nm SMYL system (Supra 577Y Subliminal Laser System; Quantel Medical, ClermontFerrand, France) was used for the treatment. Laser application was performed with an Area-Centralis lens (Volk Optical, Mentor, OH, USA). Micropulse laser power used in SMYL was derived for each eye from a test burn. Sub-threshold treatment was performed in the micropulse mode, using a $160-\mu \mathrm{m}$ spot diameter and a $20-\mathrm{ms}$ duration with $5 \%$ duty cycle (DC) energy. The power was initially enhanced to the minimum threshold value to 
cause a barely visible burn, then it was reduced $50 \%$ of that energy level, so that no visible or detectable retinal changes were made. FA-guided images were used to determine the area to be treated, and the laser shots were delivered on active leakage area and surrounding neighborhood one-spot-size area. If the SRF was not completely resolved in 3 months after the treatment or there was a recurrence, SMYL was repeated.

We evaluated the BCVA and CS changes as well as changes in the OCT findings (CMT, TMV, CMV, SRFH) between the baseline and follow-up examinations. We also checked the SFCT changes assessed using the EDIOCT technique and compared them with unaffected fellow eyes until the final follow-up. All measurements were made every month for all patients, but BCVA, CS, and main OCT findings are demonstrated at baseline, 3 months after treatment and at the final follow-up in this paper.

The number of recurrences after SMYL treatment and any adverse effects were recorded. Complete response was defined as complete resolution of SRF. If SRF decreased but was not totally absorbed, the treatment response was defined as incomplete resolution. Complete resolution of SRF was accepted as anatomical success. We evaluated the patients who gained 2 or more lines in BCVA as improved and any gain less than 2-line improvement as stable and any line loss of BCVA as worsened.

\section{Statistical analyses}

Statistical analyses were performed using SPSS ver. 21,0 (SPSS Inc., Chicago, IL, USA). The distribution of the data was tested for normality using the Kolmogorov-Smirnov and Shapiro-Wilk test. The SRF measurements and CS values were normally distributed, whereas BCVA (Snellen and logMAR), CMT, SFCT, CMV, TMV were not. Pre- and post-laser SRF height was analyzed using the paired Student's $t$-test. Descriptive analyses were presented using medians and interquartile ranger for the non-normally distributed and ordinal variables. Friedman tests were conducted to test whether there is a significant change in non-normally distributed variables and the other parameters were analyzed using the Wilcoxon signed-ranks test. Statistical significance was considered when $P$-value was $<0.05$.

\section{Results}

A total of thirty-nine eyes of 39 patients with a diagnosis CCSC were included into the study and treated with SMYL. Thirty-three patients were males and six females at the diagnosis with the mean age of $43.38 \pm 13.85$ years (range: 22-72). Mean duration time of symptoms before
Table 1 Initial data of participants

\begin{tabular}{ll}
\hline Mean age (years) & $43.38 \pm 13.85$ \\
Sex & \\
Female & 6 \\
Male & 33 \\
& \\
Mean duration of symptoms (months) & $14.67 \pm 2.86$ \\
Source of leakage & \\
Focal & 30 \\
Diffuse & 9 \\
& \\
Previous treatment & \\
None & 22 \\
IVRNB & 5 \\
IVB & 5 \\
PDT & 2 \\
Focal Laser & 1 \\
PDT+ IVB & 1 \\
Focal laser+IVB & 3 \\
\end{tabular}

Mean post-SMYL follow-up period (months)

$19.82 \pm 0.42$

Abbreviations: IVRNB, intravitreal ranibizumab; IVB, intravitreal bevacizumab; PDT, photodynamic therapy; SMYL, sub-threshold micropulse yellow laser.

SMYL treatment was $14.67 \pm 2.86$ months (range: 4-60 months). In 10 eyes (25\%) the duration of the diseases was $<6$ months and in 29 eyes $(75 \%)$ it was more than 6 months. There were 17 patients who had received previous various treatment before SMYL treatment.

Table 1 shows initial data of all patients.

The mean post-SYML treatment follow-up period was $19.82 \pm 0.42$ months (range: 16-27 months). The mean number of treatment sessions, including initial treatment and re-treatment was 1.4 (range: 1-4). The laser power used in our study ranged from 280 to $600 \mathrm{~mW}$ (mean: 44953 ) and the average number of burns placed was 40633 (280-746). There were 30 eyes (76.9\%) with single leaks and $9(23.1 \%)$ with areas of multiple leaks on FA.

Median BCVA before treatment was 0.40 Snellen (0.40 $\log$ MAR and mean BCVA: $0.44 \pm 0.20$ Snellen). Median BCVA at 3 months after treatment was 1.0 (0.0 logMAR and mean $0.84 \pm 0.22$ Snellen). The median BCVA at final follow-up was $1.00(0.0 \log$ MAR and mean $0.87 \pm 0.20$ Snellen). The BCVA was improved statistically significant at 3 months $(P<0.01)$ and at the final follow-up $(P<0.01)$ after the treatment. Of the 39 eyes, 32 eyes had 2 or more lines improvement, 3 eyes had one line improvement and 4 eyes had retained vision and accepted as stable at the end of 3 months. BCVA was improved in 35 eyes (89.7\%), stable in 4 eyes $(10.3 \%)$, worsened in none of the eyes at the final follow-up. The mean initial $\log$ CS was $1.32 \pm 0.28$, whereas at 3 months mean $\log C S$ was $1.78 \pm 0.12$; it was $1.81 \pm 0.14$ at the last visit $(P<0.05)$. 
All eyes showed a significant decrease in the SRF on SD-OCT; the average decrease in fluid height was $88.7 \%$ $(P<0.001)$ by the end of three months. The mean resolution time of SRF was 2.10 months (range: 1-4 months). In 10 eyes with disease duration $<6$ months, mean SRF resolution time was 2.33 months and 29 eyes with diseases duration more than 6 months, mean SRF resolution time was 2.03 months. These differences were not statistical significant $(P=0.45)$. At 3 months, 32 eyes $(82.05 \%)$ had complete resolution, and out of 39 eyes, 36 (92.3\%) had complete SRF resolution at the final follow-up. At the final follow-up, residual SRF was present in three eyes $(7.7 \%)$ in which re-treatment was performed. These three eyes had multiple leaks on FA and had longer duration time, 8, 24, and 60 months, respectively. The mean value of SRFH was $182 \mu \mathrm{m}$ before laser treatment, whereas it was only $10 \mu \mathrm{m}$ at last visit for three patients. Thus, SRFH was decreased by $93.8 \%$ after treatment.

The median CMT before treatment was $369 \mu \mathrm{m}$, in comparison to $256 \mu \mathrm{m}$ after 3 months and $250 \mu \mathrm{m}$ at the last visit, respectively $(P<0.01)$. The initial median $\mathrm{CMV}$ and TMV decreased from 0.30 and $9.86 \mathrm{~mm}^{3}$ to 0.20 and $8.90 \mathrm{~mm}^{3}$ at the 3 months, respectively, and to 0.19 and $8.76 \mathrm{~mm}^{3}$ at the final follow-up, respectively (Figures 1 and 2 shows a patient's FA and OCT finding). At the last visit, among the patients who had complete SRF resorption, the group of patients who had CMV levels higher than $0.2 \mathrm{~mm}^{3}$ had better BCVA (mean BCVA was $0.92 \pm 0.04)$ compared the group of patients had CMV levels $<0.2 \mathrm{~mm}^{3}$ (mean BCVA was $0.78 \pm 0.14$ ). However, these differences were not statistically significant $(P=0.064)$. In addition, we recorded longer duration time of symptoms with eyes who had CMV levels $<0.2 \mathrm{~mm}^{3}$.

The initial median SFCT in the affected eyes was $364 \mu \mathrm{m}$, which was significantly higher than the fellow eyes (unaffected eyes-321 $\mu \mathrm{m}$ ). The median SFCT values in affected eyes were $350 \mu \mathrm{m}$ at 3 months and $342 \mu \mathrm{m}$ at the last visit. There were statistical differences between initial, 3 months and last follow-up values in term of SFCT decrease $(P<0.01)$. The final SFCT differences between affected and unaffected eyes were not statistically significant $(P>0.01)$. Table 2 shows the changes of BCVA and OCT findings.

There were nine patients who had recurrent CSCR, with a mean recurrence time of 6 months (range: 4-8 month). All recurrent patients received re-treatment. Six of them responded completely, but three had residual SRF at the final visit. In addition, all recurrent patients had multiple leaks. Mean age of recurrent patients $(50.41 \pm 11.8$ years $)$ was higher than non-recurrent patients $(40.25 \pm 13.72)$ and this difference was statistically significant $(P=0.032)$. There were no reported adverse events during follow-up.

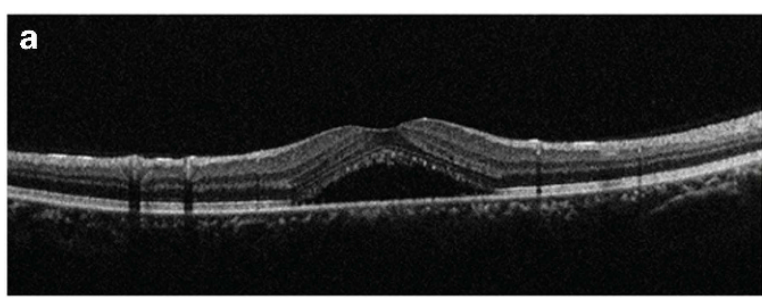

b
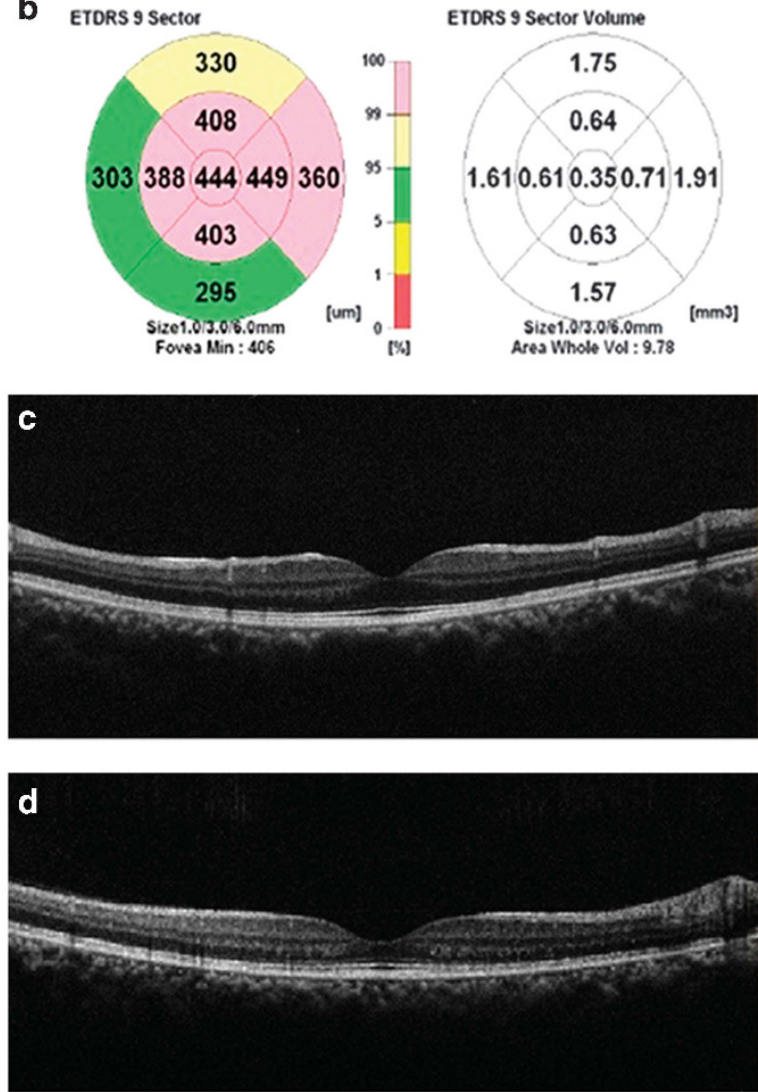

e etORs9sector

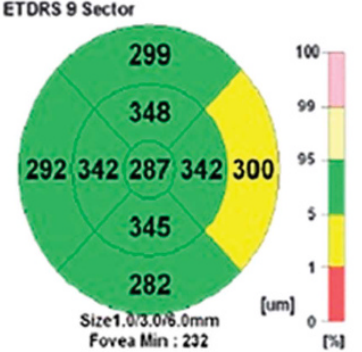

ETORS 9 Sector Volume

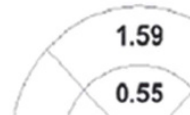

1.550 .540 .230 .541 .59

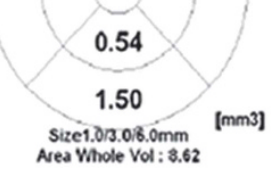

Figure 1 Thirty-two years old, female patient with nonresolving central serous chorioretinopathy of the right eye. (a) OCT image is showing neurosensory macular detachment at initial examination. (b) SRF with the corresponding CMT of $444 \mu \mathrm{m}, \mathrm{CMV}: 0.35 \mathrm{~mm}^{3}$, TMV:9.78 $\mathrm{mm}^{3}$. (c) OCT image 2 months after $577 \mathrm{~nm}$ SMYL treatment with complete resolution of the SRF. (d) OCT image 17 months after $577 \mathrm{~nm}$ SMYL treatment and complete resolution was maintained. (e) At final follow-up (17th month) CMT of $287 \mu \mathrm{m}, \mathrm{CMV}: 0.23 \mathrm{~mm}^{3}$, TMV: $8.62 \mathrm{~mm}^{3}$. 


\section{Discussion}

In CSCR, the ideal timing and treatment option remains to be determined. Although serous retinal detachment may resolve spontaneously, CSCR classically affects working middle-age individuals, whose ability to work may be compromised by the associated visual dysfunction. The presence of chronic SRF can lead to photoreceptor death, which can result in permanent visual loss and diffuse RPE damage. ${ }^{7,8,20}$ However, it is not known which patient will become chronic because the
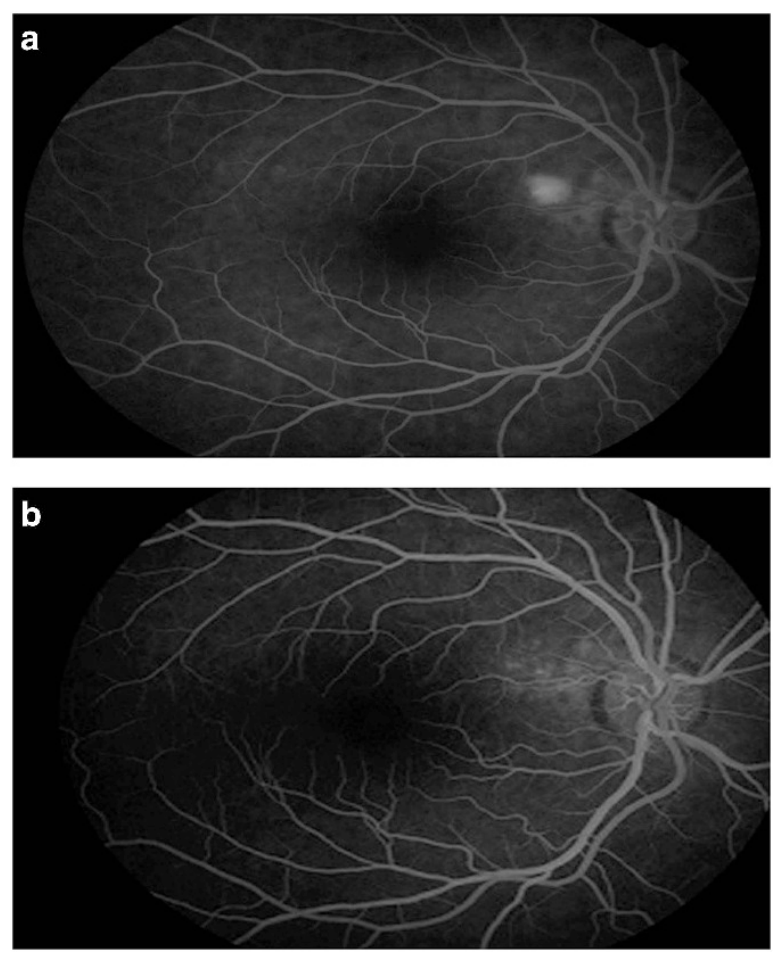

Figure 2 (a) Single leakage on FA before the treatment. (b) FA image at final follow-up (at 17th month). CMT, central macular thickness; CMV, central macular volume; FA, fluorescein angiography; OCT, optical coherence tomography; SRF, subretinal fluid; TMV, total macular volume. pathophysiology is still not clearly understood.

Abnormalities of the choroidal circulation, impaired autoregulation, and dysfunction of RPE barrier and pumping are believed to have an important role in the pathophysiology of this entity. Choroidal congestion and hyperpermeability have been frequently described in this disease. ${ }^{21,22}$ For this reason, it is referred to as part of the newly defined pachychoroid diseases group. ${ }^{23,24}$

Recently, the focus has been on treatment methods that will restore the proper pump function of RPE cells and safely reduce the choroidal vascular permeability. ${ }^{20,25}$

The $577 \mathrm{~nm}$ SMYL is designed to target the RPE while minimizing the negative thermal effects on the neural retina and deeper structures. These modality is termed 'photostimulation,' as opposed to 'photocoagulation'. ${ }^{26}$ Studies conducted to understand cellular responses to subtreshold, sublethal hypertermia showed significant increase in heat shock protein (HSP) transcription. HSPs are a group of ubiquitous, well-described proteins that are expressed in response to cellular stress. ${ }^{27}$ Acting as chaperone proteins, HSPs can assist in the refolding of denaturated proteins and inhibit inappropriate protein aggregation. In addition, HSPs interact with and stabilize the cytoskeleton and help to maintain the cellular structure. The HSP70 in particular is known to be expressed in conditions of thermal, ischemic and oxidative stress. Moreover, HSPs have activity against apoptotic pathways and inflammation. ${ }^{28}$

In this study, 577-nm SMYL for CCSC showed good long-term clinical effects, improving BCVA and decreasing CMT. BCVA was improved in 35 eyes (89.7\%), stable in 4 eyes $(10.3 \%)$ at the final follow-up. CMT was decreased from 369 to $256 \mu \mathrm{m}$ at 3 months and to $250 \mu \mathrm{m}$ at the last visit. These results are comparable with those of studies that used 577-nm SMYL treatment, such as that of Abd Elhamid, who reported that BCVA was improved in nine eyes $(60 \%)$ stable in six $(40 \%)$ at 6 months after SMYL treatment. In this study, residual SRF was present in two eyes after 6 months using 577-nm SMYL with $10 \%$ DC. ${ }^{29}$ The 6-week results of another recent study using 577-nm

Table 2 Data summary of non-parametric values statistical analyses

\begin{tabular}{|c|c|c|c|c|c|c|c|}
\hline & \multirow[t]{2}{*}{$\mathrm{P}$} & \multicolumn{2}{|c|}{ Initial } & \multicolumn{2}{|c|}{3 month } & \multicolumn{2}{|c|}{ Final } \\
\hline & & Median & 25-75 per & Median & 25-75 per & Median & 25-75 per \\
\hline BCVA & $<0.001$ & 0.40 & $0.30-0.60$ & 1.00 & $0.70-1.00$ & 1.00 & $0.80-1.00$ \\
\hline BCVA (LogMar) & $<0.001$ & 0.40 & $0.22-0.52$ & 0 & $0-0.15$ & 0 & $0-0.10$ \\
\hline CMT & $<0.001$ & 369 & $344-437$ & 256 & $222-267$ & 250 & $220-260$ \\
\hline CMV & $<0.001$ & 0.30 & $0.28-0.36$ & 0.20 & $0.18-0.21$ & 0.19 & $0.18-0.21$ \\
\hline TMV & $<0.001$ & 9.86 & $9.68-10.08$ & 8.90 & $8.68-9.02$ & 8.76 & $8.58-8.96$ \\
\hline SFCT & $<0.001$ & 364 & $341-396$ & 350 & $336-380$ & 342 & $330-370$ \\
\hline
\end{tabular}

Abbreviations: BCVA, best-corrected visual acuity; CMT, central macular thickness; CMV, central macular volume (mm ${ }^{3}$ ); SFCT, subfoveal choroidal thickness $(\mu \mathrm{m})$; TMV, total maular volume $\left(\mathrm{mm}^{3}\right)$.

Data are presented as median (interquartile range). 
SMYL with 5\% DC in 42 eyes showed that 23 eyes had one or more line improvements, whereas 33 were regarded as having responded to treatment. ${ }^{30} \mathrm{CS}$ was improved with $577 \mathrm{~nm}$ SMYL treatment. CS is important because it provides valuable information, independent of VA. Furthermore, it is an important predictor of realworld performance and may be useful for monitoring ophthalmic treatment and detecting disease, especially in CSC. ${ }^{31}$ Low CS levels may be present even in spontaneously resorbed or those responsive to treatment. SML application might be another beneficial point in this cases.

The lack of a well-defined titration procedure and a wide variety of settings in various clinical trials is reflected in the variable results with this technology exhibited herein. As far as we know, this is the only study using $577 \mathrm{~nm}$ SMYL with 5\% DC mode delivered only on FA leakage points. In this study, we showed the efficacy and safety of 577-nm SMYL with 5\% DC energy in CCSC treatment. Kim et $a l^{19}$ and Yadav et $a l^{32}$ reported that 577-nm SMYL for CCSC showed good short-term clinical effects, improving BCVA and CMT, with 15 and 10\% DC, respectively. These studies had smaller amount of participants compared to our case series.

SML technology can be performed with either 810-nm or 577-nm wavelength lasers. The 577-nm yellow laser light provides maximum absorption by both of oxyhemoglobin and melanine. ${ }^{33}$ This leads to energy being concentrated in a smaller volume, which in turn allows for a reduction in power and shortened pulse duration. It also has negligible xanthophyll absorption, which allows more secure treatment close to the fovea. ${ }^{34}$ There are several studies using 810-nm diod SML for CSC treatment, but their average laser power were higher than mean laser power used in this study. ${ }^{17,35}$ This may emphasize that yellow wavelength laser is better absorbed by RPE and hemoglobin, thus achieving the target effects at lower power than 810-nm diode SML modalities. However, no study has reported complications attributed to treatment, except for two studies using 810-nm diode SML, which reported some pigmentary changes in the RPE that were attributed to SML treatment but only in a few cases. ${ }^{35,36}$ Review of the published studies on 'nondamaging retinal laser therapy' showed improvements in visual acuity and retinal thickness, especially for the studies that did include observation, there was a statistically significant treatment effect noted at all time points. ${ }^{37}$

Monthly follow-ups were preferred to determine the average SRF resolution time and SFCT changes as well as to detect possible side effects. The mean SRF resolution time was 2.1 months (range: $1-4$ months). This resolution time range may be an indicator when determining the anatomical recovery period and the observation time of re-treatment decision. Although this time period involves a spontaneous regression period according to some authors, who classify it as a chronic diseases after 6 months of observation, we believe that the response to treatment was relatively rapid and safe as it was correlated with SFCT decrease. As Daruich et al ${ }^{38}$ pointed, older age, higher SFCT, and the higher degree of RPE alteration at leakage sites are independent factors of longer acute CSC episodes. One of the study which compared the efficacy of SMYL and PDT, it was shown that both half-dose PDT and 577-SMYL were potent treatments for CCSC. But patients with a diseases duration of $<1$ year showed a better treatment response only after SMYL and besides they observed choroidal neovascularization and allergic reaction in PDT group. ${ }^{39}$ 577-SMYL treatment can be chosen as an alternative early treatment modality in some subgroup patients who are prone to longer episodes and chronic changes.

Another finding of our study was that after the SMYL, the SFCT were significantly decreased; there were no significant differences between the SFCT values of the affected and unaffected eyes at the last visit. Kim et al ${ }^{19}$ and Ozmert et al ${ }^{40}$ showed that choroidal thickness did not change with statistically significantly at final followup after the 577-nm SMYL treatment. These authors performed treatment over the entire area of CSC which had formerly used an OCT guided approach to cover the edematous area entirely on the OCT thickness map. We believe that the treatment of limited affected areas performed by FA-guided technique is the reason of our finding that SFCT was decreased due to balancing of nonfunctional and functional RPE cells. As this limited treatment option helps decrease choroidal thickness, it might be an applicable option for other pachychoroid diseases.

However, nine cases who had recurrences, had multiple leakage sites on FA and all nine of those patients had been previously treated with different treatment modalities listed on Table 1. Among these cases, three eyes had persistent SRF at final follow-up. Old age, diffuse RPE alterations and multiple leakage sites were the common features in these three patients. This was also reported in a recent study emphasizing younger patients responded better to therapy with $577 \mathrm{~nm}$ SMYL. ${ }^{41}$ Although our findings suggest that $577 \mathrm{~nm}$ SMYL for CCSC is effective and safe, this new laser modality may not completely prevent the recurrence of CCSC with multiple leakage in older patients.

The limitations of the study include the lack of a control group and procedure guidelines, including laser power setting, precise indications of re-treatment, a follow-up plan, and a re-treatment plan. We have detected SMYL treatment to be an effective method in the management of CCSC. There was a significant anatomical (resolution of 
SRF) and functional success (improving BCVA and CS) in the treated eyes in our study. As the response was rapid and the procedure was safe, it may be possible to offer this treatment at an earlier time to patients with a significant drop in vision, in those requiring early visual rehabilitation, those with a massive SRF, for 'one-eyed' patients or those with mandatory corticosteroid use, such as transplant patients at the early episode itself.

Treatment at an early stage may prevent recurrences and irreversible visual loss. In sum, 577-nm SMYL is a valid treatment option for CCSC.

\section{Summary}

What was known before

- Our study showed that sub-threshold micropulse yellow laser treatment is an effective method in the management of CCSC. There was a significant anatomical (resolution of subretinal fluid) and functional success (improving visual acuity and contrast sensitivity) in the treated eyes in our study.

\section{What this study adds}

- This is a new laser treatment procedure and this study include large population and long-term follow-up.

\section{Conflict of interest}

The authors declare no conflict of interest.

\section{References}

1 Haimovici R, Koh S, Gagnon DR, Lehrfeld T, Wellik S. Risk factors for central serous chorioretinopathy. Ophthalmology 2004; 111: 244-249.

2 Loo RH, Scott IU, Flynn Jr HW, Gass JD, Murray TG, Lewis ML. Factors associated with reduced visual acuity during long-term follow-up of patients with idiopathic central serous chorioretinopathy. Retina 2002; 22: 19-24.

3 Bujarborua D. Long-term follow-up of idiopathic central serous chorioretinopathy without laser. Acta Ophthalmol Scand 2001; 79: 417-421.

4 Levine R, Brucker AJ, Robinson F. Long-term follow-up of idiopathic central serous chorioretinopathy by fluorescein angiography. Ophthalmology 1989; 96: 854-859.

5 Reibaldi M, Cardascia N, Longo A, Furino C, Avitabile T, Faro S. Standard-fluence versus low-fluence photodynamic therapy in chronic central serous chorioretinopathy: a nonrandomized clinical trial. Am J Ophthalmol 2010; 149: 307-315.

6 Wong KH, Lau KP, Chhablani J, Tao Y, Li Q, Wong IY. Central serous chorioretinopathy: what we have learnt so far. Acta Ophthalmol 2016; 94: 321-325.

7 Maaranen TH, Tuppurainen KT, Mantyjarvi MI. Color vision defects after central serous chorioretinpathy. Retina 2000; 20: 633-637.

8 Koskela P, Laatikainen L, von Dickhoff K. Contrast sensitivity after resolution of central serous retinopathy. Graefe's Arch Clin Exp Ophthalmol 1994; 232: 473-476.
9 Ober MD, Yannuzzi LA, Do DV, Spaide RF, Bressler NM, Jampol LM. Photodynamic therapy for focal retinal pigment epithelial leaks secondary to central serous chorioretinopathy. Ophthalmology 2005; 112: 2088-2094.

10 Yannuzzi LA, Slakter JS, Gross NE, Spaide RF, Costa DL, Huang SJ. Indocyanine green angiography-guided photodynamic therapy for treatment of chronic central serous chorioretinopathy: a pilot study. Retina 2012; 32: 288-298.

11 Mainster MA, White TJ, Allen RG. Spectral dependence of retinal damage produced by intense light sources. J Opt Soc Am 1970; 60: 848-855.

12 McKenzie AL. Physics of thermal processes in laser-tissue interaction. Phys Med Biol 1990; 35: 1175.

13 Maruko I, Koizumi H, Hasegawa T, Arakawa H, Iida T. Subthreshold $577 \mathrm{~nm}$ micropulse laser treatment for central serous chorioretinopathy. PLoS One 2017; 12: e0184112.

14 Brancato R, Pratesi R, Leoni G, Trabucchi G, Vanni U. Histopathology of diode and argon laser lesions in rabbit retina. A comparative study. Investig Ophthalmol Vis Sci 1989; 30: 1504-1510.

15 Dorin G. Subthreshold and micropulse diode laser photocoagulation. Semin Ophthalmol 2003; 18: 147-153.

16 Dorin G. Evolution of retinal laser therapy: minimum intensity photocoagulation (MIP). Can the laser heal the retina without harming it? Semin Ophthalmol 2004; 19: 62-68.

17 Malik KJ, Sampat KM, Mansouri A, Steiner JN, Glaser BM. Low-intensity/high-density subthreshold micropulse diode laser for chronic central serous chorioretinopathy. Retina 2015; 35: 532-536.

18 Gupta B, Elagouz M, McHugh D, Chong V, Sivaprasad S. Micropulse diode laser photocoagulation for central serous chorio-retinopathy. Clin Exp Ophthalmol 2009; 37: 801-805.

19 Kim JY, Park HS, Kim SY. Short-term efficacy of subthreshold micropulse yellow laser (577-nm) photocoagulation for chronic central serous chorioretinopathy. Graefe's Arch Clin Exp Ophthalmol 2015; 253: 2129-2135.

20 Nicholson B, Noble J, Forooghian F, Meyerle C. Central serous chorioretinopathy: update on pathophysiology and treatment. Surv Ophthalmol 2013; 58: 103-126.

21 Spaide RF, Goldbaum M, Wong DWK, Tang KC, Iida T. Serous detachment of the retina. Retina 2003; 23: 820-846.

22 Tittl M, Polska E, Kircher K et al. Topical fundus pulsation measurement in patients with active central serous chorioretinopathy. Arch Ophthalmol 2003; 121: 975-978.

23 Gallego-Pinazo R, Dolz-Marco R, Gómez-Ulla F, Mrejen S, Freund KB. Pachychoroid diseases of the macula. Med Hypothesis Discov Innov Ophthalmol 2014; 3: 111-115.

24 Takkar B, Azad S, Roshan T. Optical coherence tomography angiography of shallow irregular pigment epithelial detachments in pachychoroid spectrum disease. Am J Ophthalmol 2016; 163: 193.

25 Bandello F, Lanzetta P, Furlan F, Polito A. Non visible subthreshold micropulse diode laser treatment of idiopathic central serous chorioretinopathy. A pilot study. Investig Ophthalmol Vis Sci 2003; 44: 4858-4858.

26 Koss MJ, Beger I, Koch FH. Subthreshold diode laser micropulse photocoagulation versus intravitreal injections of bevacizumab in the treatment of central serous chorioretinopathy. Eye 2012; 26: 307-314.

27 Furukawa A, Koriyama Y. A role of heat shock protein 70 in photoreceptor cell death: potential as a novel therapeutic 
target in retinal degeneration. CNS Neurosci Therap 2016; 22: 7-14.

28 Sramek C, Mackanos M, Spitler R, Leung LS, Nomoto H, Contag $\mathrm{CH}$. Non-damaging retinal phototherapy: dynamic range of heat shock protein expression. Investig Ophthalmol Vis Sci 2011; 52: 1780-1787.

29 Abd Elhamid AH. Subthreshold micropulse yellow laser treatment for nonresolving central serous chorioretinopathy. Clin Ophthalmol 2015; 9: 2277-2283.

30 Scholz P, Ersoy L, Boon CJF, Fauser S. Subthreshold micropulse laser $(577 \mathrm{~nm})$ treatment in chronic central serous chorioretinopathy. Ophthalmologica 2015; 234: 189-194.

31 Haymes SA, Roberts KF, Cruess AF, Nicolela MT, LeBlanc $\mathrm{RP}$, Ramsey MS. The letter contrast sensitivity test: clinical evaluation of a new design. Investig Ophthalmol Vis Sci 2006; 47: 2739-2745.

32 Yadav NK, Jayadev C, Mohan A, Vijayan P, Battu R, Dabir S et al. Subthreshold micropulse yellow laser $(577 \mathrm{~nm})$ in chronic central serous chorioretinopathy: safety profile and treatment outcome. Eye 2015; 29: 258-265.

33 Mainster MA. Wavelength selection in macular photocoagulation. Ophthalmology 1986; 93: 952-958.

34 Mainster MA. Decreasing retinal photocoagulation damage: principles and techniques. Semin Ophthalmol 1999; 14: 200-209.

35 Lanzetta P, Furlan F, Morgante L, Veritti D, Bandello F. Nonvisible subthreshold micropulse diode laser $(810 \mathrm{~nm})$ treatment of central serous chorioretinopathy. A pilot study. Eur J Ophthalmol 2008; 18: 934.

36 Gawcki M. Increase in central retinal edema after subthreshold diode micropulse laser treatment of chronic central serous chorioretinopathy. Case Rep Ophthalmol Med 2015; 2015: 4.

37 Wood EH, Karth PA, Sanislo SR, Moshfeghi DM, Palanker DV. Non-damaging retinal laser theraphy for treatment of central serous chorioretinopathy: what is the evidence? Retina 2017; 37: 1021-1033.

38 Daruich A, Matet A, Marchionno L, De Azevedo JD, Ambresin A, Mantel I. Acute central serous chorioretinopathy: factors influencing episode duration. Retina 2017; 37(10): 1905-1915.

39 Scholz P, Altay L, Fauser S. Comparison of subthreshold micropulse laser $(577 \mathrm{~nm})$ treatment and half-dose photodynamic therapy in patients with chronic central serous chorioretinopathy. Eye 2016; 30(10): 1371-1377.

40 Özmert E, Demirel S, Yanık Ö, Batıŏlu F. Low-fluence photodynamic therapy versus subthreshold micropulse yellow wavelength laser in the treatment of chronic central serous chorioretinopathy. J Ophthalmol 2016; 2016: 3513794.

41 Gawęcki M, Jaszczuk-Maciejewska A, Jurska-Jaśko A, Grzybowski A. Functional and morphological outcome in patients with chronic central serous chorioretinopathy treated by subthreshold micropulse laser. Graefe's Arch Clin Exp Ophthalmol 2017; 255: 2299-2306. 